\title{
Effect of aneurysm on the mechanical dissection properties of the human ascending thoracic aorta
}

\author{
Salvatore Pasta, PhD, ${ }^{\text {a,b,c,d,e }}$ Julie A. Phillippi, PhD, ${ }^{\text {d,e,f,g }}$ Thomas G. Gleason, MD, ${ }^{\text {d,e,f,g }}$ and \\ David A. Vorp, $\mathrm{PhD}^{\mathrm{b}, \mathrm{c}, \mathrm{d}, \mathrm{e}, \mathrm{g}}$
}

\begin{abstract}
Objectives: The acute dissection of an ascending thoracic aortic aneurysm (ATAA) represents a devastating separation of elastic layers occurring when the hemodynamic loads on the diseased wall exceed the adhesive strength between layers. At present, the mechanics underlying aortic dissection are largely unclear, and the biomechanical delamination properties of the aneurysmal aorta are not defined. Individuals with bicuspid aortic valve (BAV) are particularly predisposed to ascending aortic aneurysm formation, with a marked risk of aortic dissection. The purpose of this study was to evaluate and compare the dissection properties of nonaneurysmal and aneurysmal human ascending thoracic aorta from patients with BAV morphology or normal tricuspid aortic valve (TAV) morphology using biomechanical delamination testing.
\end{abstract}

Methods: The influence on the delamination strength $\left(S_{d}\right)$ of the aorta associated with BAV was compared with that in patients with TAV. After complete delamination of ATAA tissue samples, tensile tests were performed on each delaminated half for comparison of their tensile strengths.

Results: The results showed that the aneurysmal aortas with BAV and TAV have lower $\mathrm{S}_{\mathrm{d}}$ than nonaneurysmal aortas and that ATAA with BAV has a lower $S_{d}$ than that with TAV. We have found a significant difference in $S_{d}$ between longitudinal and circumferential directions of the nondiseased aorta, suggesting anisotropic dissection properties.

Conclusions: The tensile testing results suggest that the weaker intimal half of the aortic wall might fail before the outer adventitial half. Scanning electron microscope analyses suggest different failure modalities of dissection between the two morphologies, and the lower $S_{d}$ in ATAAs appears to be associated with a disorganized microstructure. BAV ATAAs have a lower $\mathrm{S}_{\mathrm{d}}$ than TAV ATAAs, suggesting a greater propensity for aortic dissection. (J Thorac Cardiovasc Surg 2012;143:460-7)

The most common lethal complication of an ascending thoracic aortic aneurysm (ATAA) is acute dissection, causing significant mortality despite advances in diagnostics and surgical management. ${ }^{1}$ The true prevalence of aortic dissection is difficult to determine, and most estimates are based on autopsy findings with evidence in $1 \%$ to $3 \%$ of all cases. The incidence of aortic dissection is believed to be 5 to 30 cases per 1 million people annually, typically presenting in elderly patients, and in males more frequently than females (ratio 3:1). Dissection occurs more typically in elderly patients in the presence of a tricuspid aortic valve (TAV)

From the Fondazione RiMED, ${ }^{\mathrm{a}}$ Palermo, Italia; and Department of Surgery, ${ }^{\mathrm{b}}$ Department of Bioengineering, ${ }^{\mathrm{c}}$ McGowan Institute for Regenerative Medicine, ${ }^{\mathrm{d}}$ Center for Vascular Remodeling and Regeneration, ${ }^{\mathrm{e}}$ Center for Thoracic Aortic Disease, ${ }^{\mathrm{f}}$ and Department of Cardiothoracic Surgery, ${ }^{\mathrm{g}}$ University of Pittsburgh, Pittsburgh, $\mathrm{Pa}$.

Funded in part by a grant from Fondazione RiMED provided to Drs Pasta and Vorp. Disclosures: Authors have nothing to disclose with regard to commercial support.

Received for publication March 18, 2011; revisions received May 25, 2011; accepted for publication July 26, 2011; available ahead of print Aug 26, 2011.

Address for reprints: David A. Vorp, PhD, Departments of Bioengineering and Cardiothoracic Surgery, University of Pittsburgh, 412 Center of Bioengineering (CNBIO), 300 Technology Drive, Pittsburgh, PA 15219 (E-mail: VorpDA@ upmc.edu).

0022-5223/\$0.00

Published by Elsevier Inc. on behalf of The American Association for Thoracic Surgery

doi:10.1016/j.jtcvs.2011.07.058 and in relatively younger patients if a bicuspid aortic valve (BAV) is present. ${ }^{2}$ The mortality rate during the first 24 to 48 hours in patients not treated surgically is as high as $74 \%{ }^{3,4}$ Even among patients treated with emergent aortic reconstruction, the operative mortality rate averages $24 \%$ worldwide. $^{5}$

Ascending aortic dissection (Stanford type A classification) begins with an intimal tear typically occurring near the sinotubular junction where the wall stresses are thought to be elevated. ${ }^{6}$ The intimal tear, often circumferential in origin, permits blood to enter the aortic wall splitting the media and progressively separating the medial plane along the axial direction of the aorta. This new "false lumen" runs parallel to the true lumen and can reenter the true lumen anywhere along the course of the dissection or exit through the adventitia, resulting in frank disruption. As the dissection extends distally, its propagation and re-entry follows unpredictable courses that can result in life-threatening ischemia of the heart, brain, abdominal viscera, spinal cord, and extremities. ${ }^{7}$

There are several risk factors predisposing patients to spontaneous dissection. Among these are severe hypertension, connective tissue disorders such as the Marfan and Ehlers-Danlos syndromes, and BAV. The congenital malformation, BAV, is distinctly associated with the 


$$
\begin{aligned}
& \text { Abbreviations and Acronyms } \\
& \text { AAA }=\text { abdominal aortic aneurysm } \\
& \text { ADV-DEL }=\text { adventitial surface and delaminated } \\
& \text { plane } \\
& \text { ATAA = ascending thoracic aortic aneurysm } \\
& \text { BAV = bicuspid aortic valve } \\
& \text { CIRC = circumferential } \\
& \text { INT-DEL }=\text { intimal surface and delaminated } \\
& \text { plane } \\
& \text { LONG = longitudinal } \\
& \mathrm{S}_{\mathrm{d}} \quad=\text { delamination strength } \\
& \text { SEM = scanning electron microscope } \\
& \mathrm{S}_{\mathrm{T}} \quad=\text { tensile strength } \\
& \mathrm{TAV}=\text { tricuspid aortic valve } \\
& \mathrm{T}_{\text {peel }} \quad=\text { peel tension }
\end{aligned}
$$

\section{METHODS \\ Human Aortic Tissue Specimens}

All human ascending thoracic aorta tissue specimens were obtained after informed consent according to the guidelines of our institutional review board and Center for Organ Recovery and Education. Segments of the control (nonaneurysmal) aorta were collected from organ donor subjects and nondissected ATAAs were obtained fresh from patients undergoing elective ascending aortic replacement at the University of Pittsburgh Medical Center. A total of 31 aortic segments (7 controls and 24 ATAAs ) from patients with an age range of 41 to 79 years and aortic diameter of 40 to $68 \mathrm{~mm}$ (24 men and 7 women) were analyzed. The ATAAs included 16 BAV and $8 \mathrm{TAV}$ segments. Table 1 summarizes the aortic diameter, patient age, and clinical data for each human aortic tissue specimen. All nonaneurysmal segments were from subjects with TAV. The recovered aortic samples were tested within 48 hours of harvesting after storing at $4^{\circ} \mathrm{C}$ in a calcium-free and glucose-free $0.9 \%$ physiologic saline solution. ${ }^{15}$

\section{Biomechanical Testing}

The harvested aortic segments were cut into long, thin, rectangular (approximately $30 \times 6-\mathrm{mm}$ ) strips, with their long axis in either longitudinal (LONG) or circumferential (CIRC) orientation with respect to that of the aorta. Strips of both orientations were generally obtained from the same aortic segment for direct comparison. To create an initial delamination plane, a delaminating incision was made with the aid of a surgical scalpel between the elastic lamellae of each specimen, parallel with the plane of the aortic wall and 8 to $12 \mathrm{~mm}$ in length (Figure $1, A$ ). Generally, the artificial delamination plane was reproducible. However, because the initial incision was made manually, there was naturally a moderate variation in terms of the exact location of the incision plane with respect to the center of the media or to the external or internal elastic lamina. The width, thickness, and length of each specimen were measured at three different locations using a dial caliper and then averaged and recorded before testing. The two free flaps of each delaminated half of the tissue specimen were mounted between the grips of an Instron tensile system (model 5542) with a $5 \mathrm{~N}$ load cell (Figure 1,B). Surfalloy jaw faces with gritty, sandpaper-like surfaces were used to avoid slipping of the specimen in the pneumatic grips. During testing, the mounted specimens were submerged in $0.9 \%$ physiologic saline solution in a BioPuls bath under a controlled temperature of $37^{\circ} \mathrm{C}$. A constant crosshead speed of $1 \mathrm{~mm} / \mathrm{min}$ was used to pull apart the two free flaps of the delamination, and the applied load and resulting displacement were recorded continuously using the Instron-packaged software (Bluehill, version 2). The two delaminated halves resulting from each delamination test (ie, the one between the intimal surface and the delaminated plane ([INT-DEL] and the one between the adventitial surface and the delaminated plane [ADV-DEL]) were stretched to failure in the uniaxial tensile testing system to evaluate the tensile strength of each. None of specimens failed for technical reason during biomechanical testing.

\section{Statistical Analysis}

The peel tension $\left(\mathrm{T}_{\text {peel }}\right)$ was calculated as the applied force normalized by the width of the specimen and was plotted as a function of the elongation (displacement) of each specimen. The mean value of $T_{\text {peel }}$ after the initial peak was taken as the delamination strength $\left(S_{d}\right)$ of the specimen. The $S_{d}$ values calculated for multiple LONG and CIRC specimens tested for a given patient were taken as the overall $\mathrm{S}_{\mathrm{d}, \text { LONG }}$ and $\mathrm{S}_{\mathrm{d}, \text { CIRC }}$ for that specific patient.

For the tensile tests, we used the approach published previously by our laboratory. ${ }^{15}$ In brief, the Cauchy stress (T) was calculated as the applied force normalized by the deformed cross-sectional area, and the stretch was calculated as the deformed length normalized by the original length of each specimen. The tensile strength $\left(\mathrm{S}_{\mathrm{T}}\right)$ was taken as the peak value of stress attained before complete specimen failure. "false lumen" for determining the propensity of either intimal flap propagation or frank disruption. The biomechanical assays were complemented by scanning electron microscope (SEM) investigations to examine the failure modality of aortic dissection. 
TABLE 1. Clinical data of patients for each aortic tissue used for biomechanical testing

\begin{tabular}{|c|c|c|c|c|c|c|}
\hline Type & $\begin{array}{c}\text { Aneurysm } \\
\text { diameter }(\mathbf{m m})\end{array}$ & Age (y) & $\begin{array}{c}\text { Wall } \\
\text { thickness (mm) }\end{array}$ & $\begin{array}{c}\begin{array}{c}\text { History of } \\
\text { hypertension }\end{array} \\
\end{array}$ & $\begin{array}{c}\text { Aortic } \\
\text { stenosis }\end{array}$ & $\begin{array}{c}\text { Aortic } \\
\text { insufficiency }\end{array}$ \\
\hline Nonaneurysmal & & 59 & 2.0 & & & \\
\hline Nonaneurysmal & & 62 & 1.6 & Yes & & \\
\hline Nonaneurysmal & & 65 & 1.9 & & & \\
\hline Nonaneurysmal & & 55 & 2.3 & & & \\
\hline Nonaneurysmal & & 44 & 2.5 & & & \\
\hline Nonaneurysmal & & 28 & 2.0 & & & \\
\hline Nonaneurysmal & & 54 & 2.1 & & & \\
\hline Average & & $52 \pm 12$ & $2.0 \pm 0.3$ & & & \\
\hline Aneurysmal with BAV & 49 & 48 & 1.7 & & & Moderate \\
\hline Aneurysmal with BAV & 49 & 51 & 1.9 & & Severe & Severe \\
\hline Aneurysmal with BAV & 57 & 41 & 1.7 & Yes & & \\
\hline Aneurysmal with BAV & 50 & 66 & 1.7 & & Severe & \\
\hline Aneurysmal with BAV & 50 & 61 & 1.9 & Yes & Severe & Moderate \\
\hline Aneurysmal with BAV & 46 & 56 & 1.8 & & Severe & \\
\hline Aneurysmal with BAV & 53 & 42 & 1.9 & & & Mild \\
\hline Aneurysmal with BAV & 50 & 41 & 1.9 & Yes & & Moderate \\
\hline Aneurysmal with BAV & 50 & 43 & 1.8 & Yes & & \\
\hline Aneurysmal with BAV & 68 & 59 & 1.9 & & & Severe \\
\hline Aneurysmal with BAV & 52 & 52 & 1.6 & & & \\
\hline Aneurysmal with BAV & 50 & 48 & 2.1 & & Severe & \\
\hline Aneurysmal with BAV & 48 & 62 & 2.0 & & & \\
\hline Aneurysmal with BAV & 50 & 47 & 2.0 & & & Mild \\
\hline Aneurysmal with BAV & 48 & 59 & 1.7 & & Severe & \\
\hline Aneurysmal with BAV & 46 & 69 & 1.9 & & Severe & \\
\hline Average & $51 \pm 5$ & $53 \pm 9$ & $1.8 \pm 0.1$ & & & \\
\hline Aneurysmal with TAV & 65 & 72 & 1.9 & & & Moderate \\
\hline Aneurysmal with TAV & 48 & 72 & 1.9 & Yes & & Severe \\
\hline Aneurysmal with TAV & 65 & 53 & 2.3 & Yes & & Severe \\
\hline Aneurysmal with TAV & 54 & 53 & 2.0 & Yes & & Severe \\
\hline Aneurysmal with TAV & 56 & 56 & 2.1 & & & Moderate \\
\hline Aneurysmal with TAV & 52 & 73 & 2.0 & Yes & & Mild \\
\hline Aneurysmal with TAV & 54 & 67 & 2.0 & Yes & & Mild \\
\hline Aneurysmal with TAV & 45 & 66 & 2.3 & Yes & & Mild \\
\hline Average & $55 \pm 7$ & $64 \pm 9$ & $2.0 \pm 0.2$ & & & \\
\hline
\end{tabular}

Data are mean \pm standard deviation. Blank entry indicates variable was not present in the patient. $B A V$, Bicuspid aortic valve; $T A V$, tricuspid aortic valve.

One-way analysis of variance, followed by the Holm-Sidak post hoc test for all pair-wise comparisons, was performed using SigmaPlot software (SYSTAT Software, Chicago, Ill) to determine the significance among the groups. Data are presented as the mean \pm standard error of the mean.

\section{SEM Imaging}

To observe the changes in tissue microstructure due to the propagation of the dissection, the surfaces of delaminated halves of specimens from each group were examined under a SEM at $3 \mathrm{kV}$. The aortic tissues were fixed in $2.5 \%$ glutaraldehyde for 1 hour, dehydrated in a graded series of ethanol/water solutions, dried, and then sputter coated with gold. The orientation of each specimen inside the microscope was aligned to that of the dissection propagation before imaging.

\section{RESULTS}

Figure 2 shows representative delamination curves for three separate LONG and CIRC strips cut from an individual ATAA with BAV. The initial "ramp" phase of the loading curve corresponds to stretching of the peel arms, and the jagged plateau region shows the slow and controlled propagation of the dissection. The oscillation is typical for these experiments and is often referred in the rubber mechanics data as unstable or "stick-slip" tearing (ie, the delamination does not propagate at a steady rate but is arrested and re-initiated at irregular intervals).

Figure 3, $A$ illustrates the comparison of $\mathrm{S}_{\mathrm{d}}$ between the nonaneurysmal and ATAA with BAV and TAV in both LONG and CIRC directions. A significant difference was noted for the delamination strength (ie, the resistance to aortic dissection) in both LONG and CIRC directions of the nonaneurysmal aorta $\left(\mathrm{S}_{\mathrm{d}, \mathrm{LONG}}=149.0 \pm 7.6\right.$ and $\mathrm{S}_{\mathrm{d}, \mathrm{CIRC}}=126.0 \pm 6.6, \mathrm{n}=7$ ) with respect to that of the aneurysmal aorta with BAV $\left(\mathrm{S}_{\mathrm{d}, \mathrm{LONG}}=100.0 \pm 4.1\right.$ and $\left.\mathrm{S}_{\mathrm{d}, \mathrm{CIRC}}=88.4 \pm 4.1, \mathrm{n}=16\right)$ and with respect to that of the aneurysmal aorta with $\mathrm{TAV}\left(\mathrm{S}_{\mathrm{d}, \mathrm{LONG}}=116.8 \pm 6.1\right.$ 


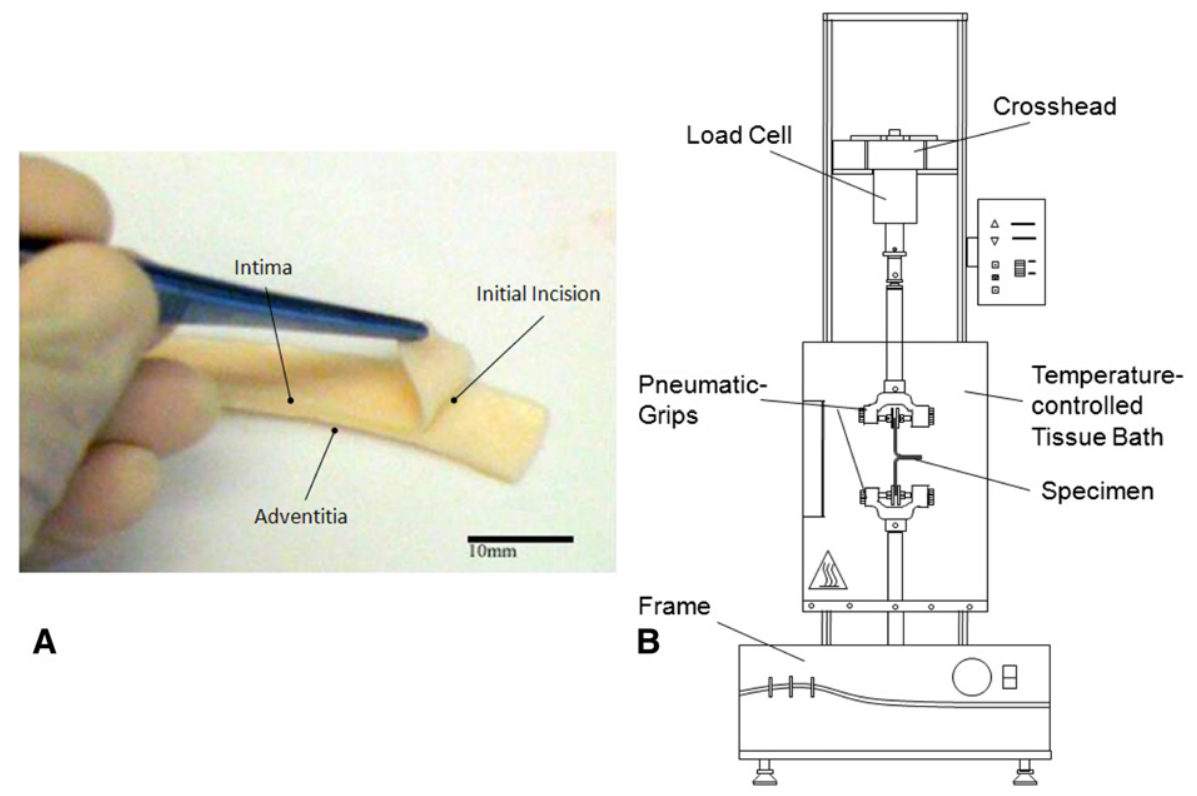

FIGURE 1. A, Photograph of representative specimen for delamination test showing through-thickness incision for creating initial dissection plane. $\mathrm{B}$, Schematic experimental setup of delamination testing.

and $S_{\mathrm{d}, \mathrm{CIRC}}=109.1 \pm 5.2, \mathrm{n}=8$ ). Moreover, the significant difference between the LONG and CIRC strengths for the nonaneurysmal group indicates anisotropic (ie, directionally dependent) dissection properties of the human ascending thoracic aorta. However, this anisotropy is not displayed by the aneurysmal aorta because both BAV and TAV groups do not present statistical differences among the orientations. The delamination strength of aneurysmal aortic tissues with BAV was significantly lower than TAV in both LONG and CIRC orientations.

We recognize that the $S_{d}$ could be affected by patient age or aneurysm diameter. To determine whether the difference in $S_{d}$ between groups was age dependent, the $S_{d}$ data for the nonaneurysmal and BAV groups as a function of patient age were fit by linear regression analysis to calculate two new sets of extrapolated $\mathrm{S}_{\mathrm{d}}$ for nonaneurysmal and BAV, respectively, at the age of each of the patients with TAV (Figure 3, $B)$. To assess whether the difference in $S_{d}$ between aneurysmal groups was diameter dependent, the $S_{d}$ data for the BAV group as a function of the aneurysm diameter were fit by linear regression to obtain a new set of extrapolated $S_{d}$ for BAV at the aneurysm diameter of each of the TAV patients (Figure 3,C). For the latter analysis, the comparison was performed only between aneurysmal groups because the $\mathrm{S}_{\mathrm{d}}$ cannot be extrapolated for the nonaneurysmal aorta as a function of aortic diameter. The results suggest that even with corrections of age or aneurysm diameter, the $S_{d}$ of ATAA with TAV remains greater than that of $\mathrm{BAV}_{\mathrm{ext}}$ (Figure 3, B,C).

Figure 3, $D, E$ shows the comparison of $\mathrm{S}_{\mathrm{T}}$ for the nonaneurysmal and aneurysmal tissues for both INT-DEL and
ADV-DEL halves after the delamination tests in the LONG and CIRC orientations. In all cases, the ultimate tensile strength of the CIRC strips was found to be greater than that for LONG specimens and the INT-DEL half is significantly weaker than the ADV-DEL half. It should also be noted that the $S_{T}$ of aneurysms with BAV is greater than that of TAV although not significantly different. This trend is opposite of that observed for the delamination testing.

The related SEM images of the dissected surfaces for the healthy aorta revealed that the delamination in the LONG direction creates a remarkably "rougher" surface compared with the surfaces from the CIRC specimens (Figure 4, A). Similar failure surfaces were found for either type of aneurysm; however, they appeared rougher than those of the normal aorta (Figure 4,A). A "fiber bridging" failure modality, which occurs when the dissection switches from one fiber/ matrix interface to another and leaves behind the unbroken fibers to bridge the delamination, was observed at high magnification for both nondiseased and aneurysmal aortas (Figure 4, B).

\section{DISCUSSION}

This study was performed to explore the delamination properties of the human ascending aorta to improve our understanding of the mechanics underlying aortic dissection in patients with ATAAs and to compare these properties in patients with BAV and TAV. We also evaluated the mechanical integrity of the outer versus inner half of the dissected aorta to assess the relative probability of exit through the adventitia (disruption) versus propagation of the dissection flap, respectively, after the onset of 

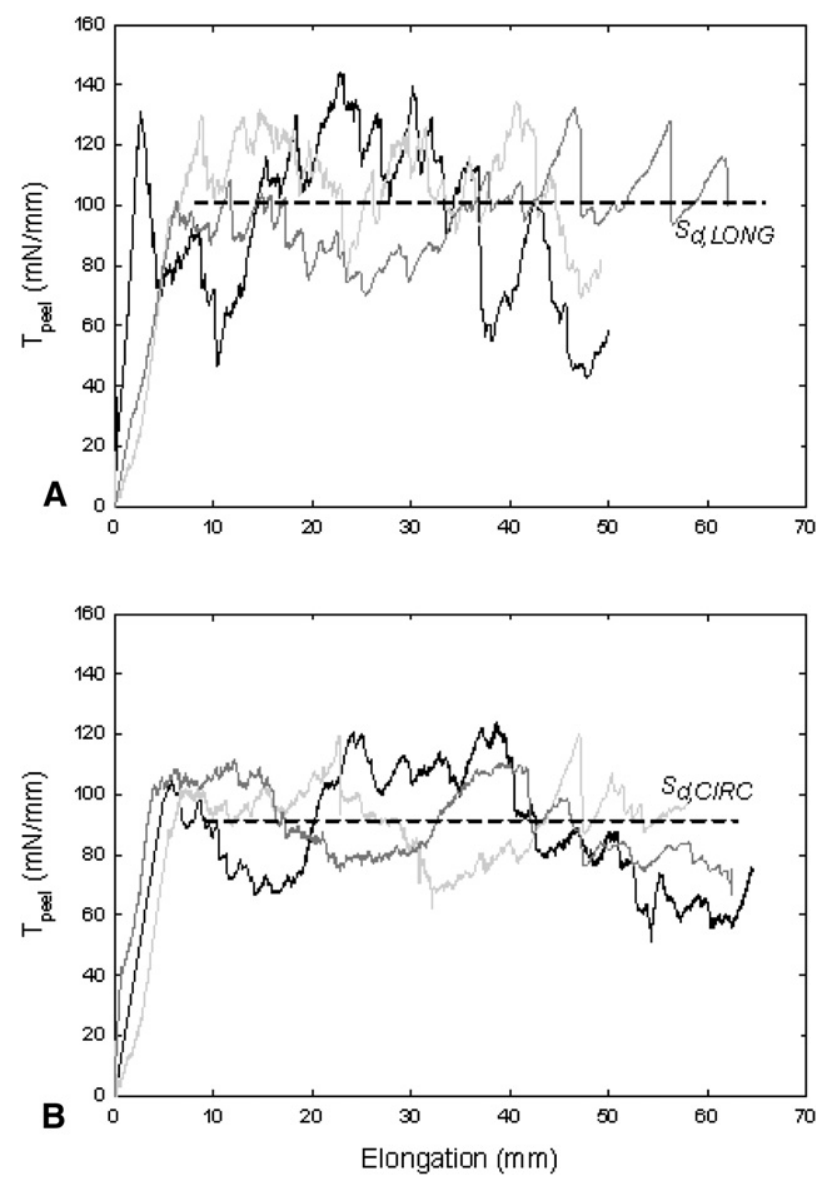

FIGURE 2. Delamination profiles for A, three longitudinal (LONG) and $\mathrm{B}$, three circumferential $(C I R C)$ strips cut from same bicuspid aortic valve $(B A V)$ aneurysm harvested from 56-year-old male patient with aortic diameter of $46 \mathrm{~mm}$. Dashed lines represent average of mean values of peel tension $\left(T_{\text {peel }}\right)$ for all LONG and CIRC strips and taken as delamination strength $\left(S_{d}\right)$ in LONG and CIRC directions for patient, respectively.

dissection. Finally, the failure mechanisms during dissection were optically investigated. Our results suggest that the risk of dissection is greater in the aneurysmal aorta compared with the nonaneurysmal aorta and is intrinsically greater for ATAAs with BAV than those with TAV. To our knowledge, these findings have never been reported. Sommer and colleagues ${ }^{16}$ reported similar dissection properties for the human abdominal aorta. However, their study was limited by a relatively small number of specimens and because aortic dissection rarely occurs in the abdominal aorta, except in the case of propagation of thoracic aortic dissection.

The delamination curves (Figure 2) revealed an oscillation of $\mathrm{T}_{\text {peel }}$ about a mean "plateau" value similar to the results found for tearing tests of the pig descending aorta ${ }^{17}$ and peeling tests of the human abdominal aorta. ${ }^{16}$ This suggests that the dissection does not propagate at a steady rate but arrests and reinitiates at somewhat regular intervals. ${ }^{18}$ The force necessary to drive the dissection varies widely from a maximum at delamination initiation to a minimum at delamination arrest. This mode of failure is also supported by the large number of broken elastin fibers found in the SEM results (Figure 4, $B$ ) and is consistent with the fiber bridging failure modality. ${ }^{19}$ In this manner, the elastin fibers between halves might bear high stretch values during delamination testing, with a consequent increase in the $T_{\text {peel }}$ magnitude. Their subsequent failure causes a rapid decrease in $\mathrm{T}_{\text {peel }}$ owing to the reduced resistance to delamination, and this process repeats in intervals as the delamination propagates. Delamination was observed during the testing to propagate entirely within the medial layer of the aortic tissue specimens.

The significantly lower resistance to aortic dissection for either type of aneurysm compared with that of the nonaneurysmal aorta (Figure 3,A) demonstrates that patients with thoracic aneurysms are more prone to aortic dissection. We reported similar findings in tensile strength, suggesting that the risk of rupture in ATAAs is $30 \%$ greater than that in nonaneurysmal ascending aortas. ${ }^{20}$ Iliopoulos and colleagues $^{21}$ reported lower LONG tensile strength with aneurysm enlargement and suggested that this might be a cause of aortic dissection in ATAAs. That we found a $S_{d, C I R C}$ significantly lower than the $S_{\mathrm{d}, \mathrm{LONG}}$ is consistent with the notion that the pathogenesis of aortic dissection is initiated by a transverse intimal tear on most of tear morphology seen clinically. ${ }^{6}$ Anisotropic dissection properties of the nonaneurysmal human ascending thoracic aorta are deduced by the significant difference of the delamination strength in LONG and CIRC orientations (Figure 3, A). In contrast, aneurysmal disease leads to isotropic behavior of the aorta probably because of a more disorganized microstructure (Figure 3, A). The most striking finding is the difference in delamination propensity among BAV ATAAs compared with TAV ATAAs (Figure 3, A), suggesting a greater risk of dissection among patients with BAV. Furthermore, our deduction that age and aneurysm diameter are not key factors for aortic dissection in patients with thoracic aneurysms (Figure 3, B,C) suggests that the greater risk of dissection in subjects with BAV is related to lower delamination properties that might be caused by predisposing structural disorders. For example, the lower $S_{d}$ in BAV tissue might be due to inherent deficits in smooth muscle cell response to oxidative stress. $^{22,23}$

Our results demonstrated a weaker $\mathrm{S}_{\mathrm{T}}$ of the intimal half of the aortic wall (ie, the INT-DEL half; Figure $3, D, E$ ), imparting an apparent risk of dissection propagation. Exit through the outer residual layer (ie, ADV-DEL half), causing frank aortic disruption is less common than dissection propagation, and this might be explained by the relatively stronger $S_{T}$ of the adventitial half of the aortic wall in this study. Similar findings of the disparate strength of nonaneurysmal arterial layers were found by Holzapfel and colleagues. $^{24}$ 

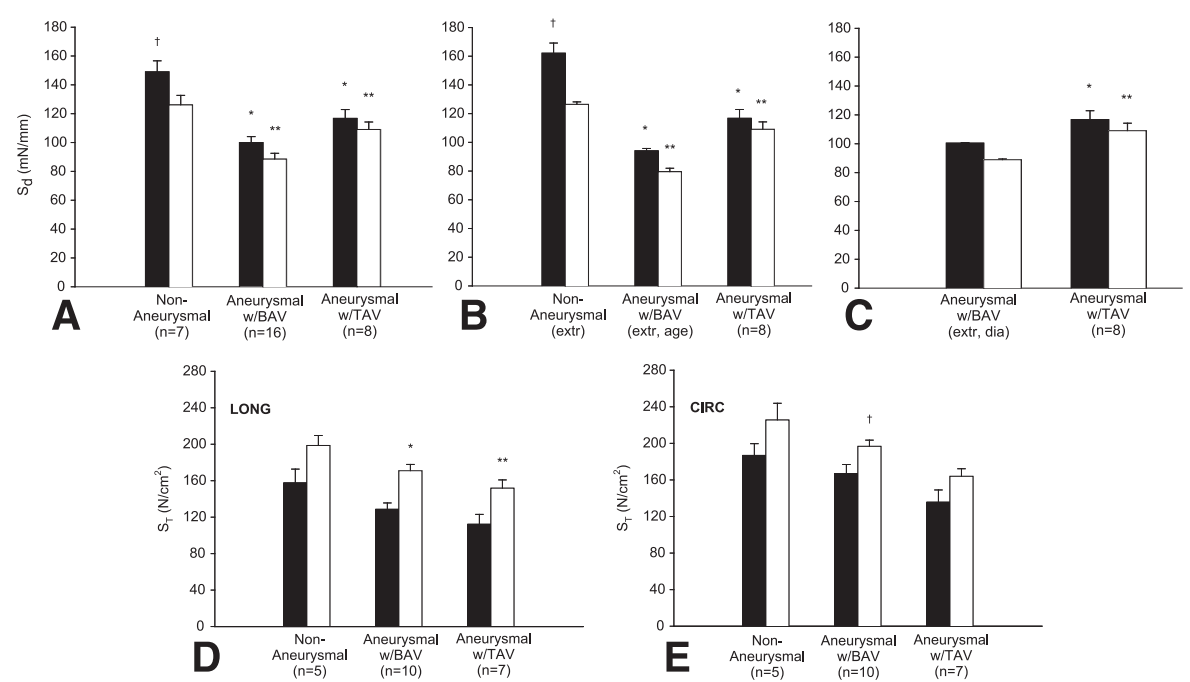

FIGURE 3. A, Delamination strength in both longitudinal (LONG) (black squares) and circumferential (CIRC) (white squares) orientations. *Significantly different from LONG nonaneurysmal aorta $(P<.05)$. **Significantly different from CIRC nonaneurysmal aorta $(P<.05)$. ${ }^{\dagger}$ Significantly different from CIRC nonaneurysmal aorta $(P<.05)$. B, Delamination strength of aneurysmal aorta with bicuspid aortic valve $\left(B A V_{\text {ext, age }}\right)$ extrapolated $($ calculated) at age of each of patients with tricuspid aortic valve (TAV) in both LONG (black squares) and CIRC (white squares) directions for $\mathrm{n}=8$ specimens. *Significantly different from LONG nonaneurysmal aorta $(P<.05) . * *$ Significantly different from CIRC nonaneurysmal aorta $(P<.05)$. ${ }^{\dagger}$ Significantly different from CIRC nonaneurysmal aorta $(P<.05)$. C, Delamination strength of aneurysmal aorta with BAV $\left(B A V_{\text {ext, dia }}\right)$ extrapolated at aneurysm diameter of each patient with TAV in both LONG (black squares) and CIRC (white squares) directions for $\mathrm{n}=8$ specimens. *Significantly different from LONG aneurysmal aorta with $\mathrm{BAV}_{\mathrm{ext}}(P<.05)$. **Significantly different from CIRC aneurysmal aorta with $\mathrm{BAV}_{\text {ext }}(P<.05)$. D, Tensile strength in LONG and E, CIRC directions for intimal surface and delaminated plane (INT-DEL) halves (black squares) and adventitial surface and delaminated plane (ADV-DEL) halves (white squares). *Significantly different from INT-DEL LONG BAV ascending thoracic aortic aneurysm $(A T A A)(P<.001)$. **Significantly different from INT-DEL LONG BAV ATAA $(P=.016)$. ${ }^{\dagger}$ Significantly different from INT-DEL CIRC TAV ATAA $(P=.024)$.

The SEM imaging results are consistent with the anisotropic dissection properties of the nonaneurysmal ascending aorta. The elastin and collagen fibers are oriented mainly in the circumferential direction in the nonaneurysmal aortic wall and, as a result, might provide a greater resistance to aortic dissection in the LONG direction. For the nonaneurysmal aorta, the creation of a rougher dissection surface might explain both greater mean and variance in $S_{d}$ of LONG strips compared with those oriented in the CIRC direction (Figure 2). Dissection in the LONG direction frequently crosses the elastic layers and that in the CIRC strips propagate mainly between adjacent elastic laminae (Figure 4, A), resulting in a "flat" broken surface, such as found by Sommer and colleagues ${ }^{16}$ for the nonaneurysmal human abdominal aorta. Moreover, the fracture surfaces of ATAAs with BAV and TAV appear rougher than those for the normal aorta (Figure 4, A), possibly owing to the more disorganized microstructure caused by the pathology. Disorganization of elastin and collagen fibers owing to the pathology appears to effect the mechanical properties of ATAAs. For the aneurysmal aorta, the creation of these rougher surfaces might explain the nonsignificant difference in $S_{d}$ between the LONG and CIRC directions, suggesting isotropic dissection properties.

Our findings were limited because delamination testing does not accurately model the spontaneous initiation of aortic dissection that occurs in vivo. Other models of aortic dissection have been described. ${ }^{25,26}$ However, the initiation of dissection was typically forced in these studies by injecting liquid using a syringe into the media to separate the aortic layers. Additionally, the stresses that lead to dissection are likely multifactorial and could be composed of stresses resulting from blood pressure, shear stresses in the blood flow field, torsion resulting from cardiac motion or propagation of the pressure pulse, and so forth. However, the purpose of this work was not to simulate these forces, but rather to measure the resistance of the tissue to stresses induced by delamination (ie, adhesive forces between the layers of the aortic wall or the delamination strength). Future work could investigate the correlation not observed in this investigation between the tissue strength and aortic diseases as aortic stenosis and regurgitation.

\section{CONCLUSIONS}

We conclude that BAV and TAV ATAAs both have significantly altered biomechanical properties compared with the normal ascending aorta, thus imparting their risk of aortic dissection. Moreover, BAV ATAAs have lower delamination strength than TAV ATAAs, rendering an apparently greater risk of aortic dissection for patients with BAV than for those with TAV. 


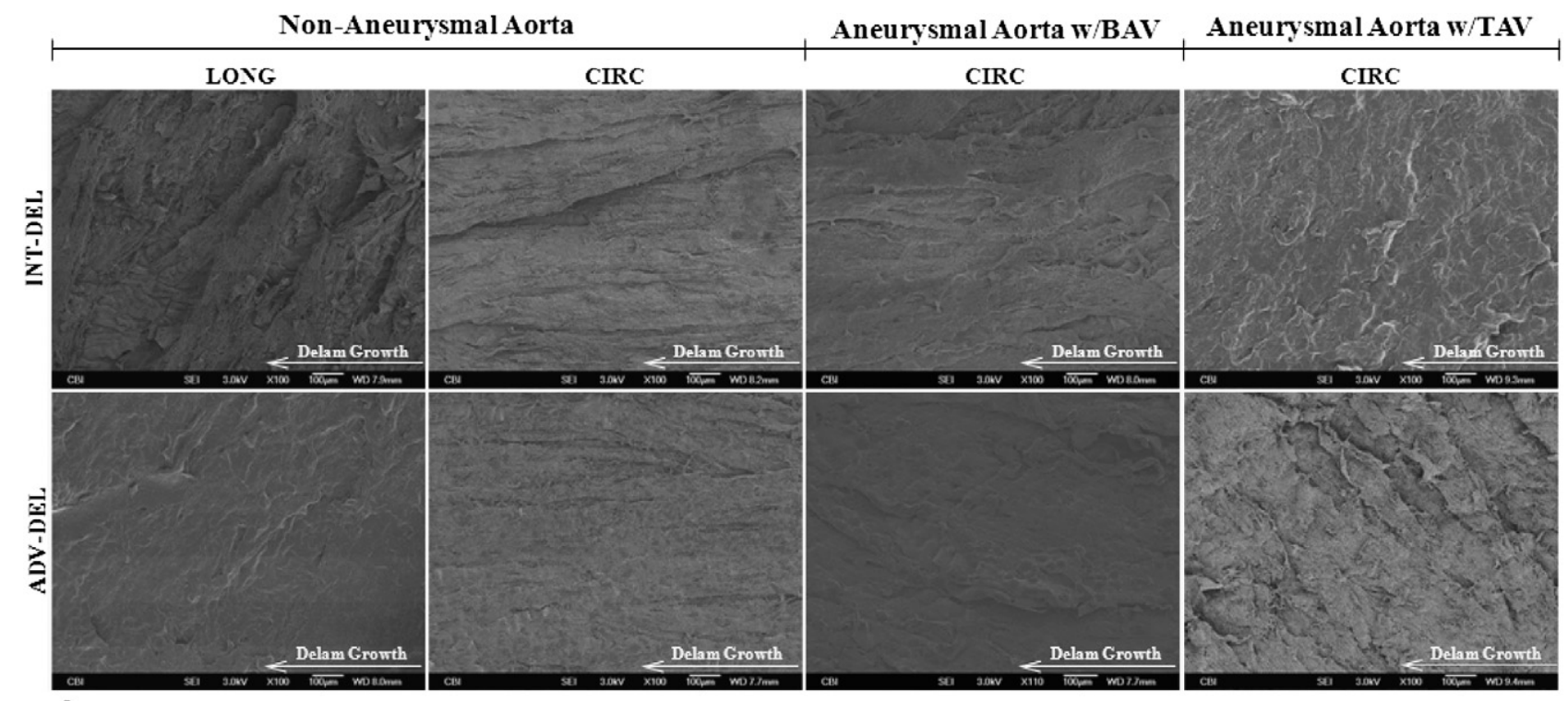

A

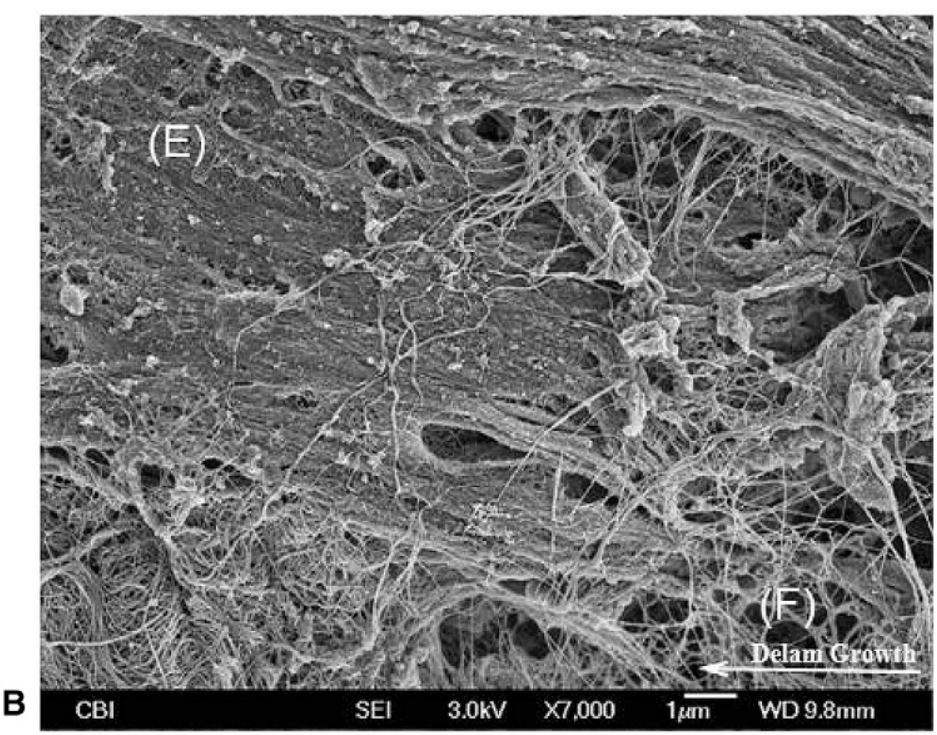

FIGURE 4. A, Representative scanning electron microscope (SEM) images of fracture surfaces of nonaneurysmal and ascending thoracic aortic aneurysm $(A T A A)$ with bicuspid aortic valve $(B A V)$ and tricuspid aortic valve $(T A V)$ in longitudinal $(L O N G)$ and circumferential $(C I R C)$ direction. B, High magnification image of CIRC TAV ATAA for intimal surface and delaminated plane (INT-DEL) half showing bundles of broken elastin fibers $(F)$ existing between elastic sheets $(E)$. Fibers act as bridge between halves in fracture modality called "fiber bridging" in delamination testing.

The authors thank Mr Jonathan Frank of the Center for Biologic Imaging of the University of Pittsburgh for his technical assistance with the SEM investigation and Mr Michael Eskay and Mr Benjamin Green for assistance with aortic tissue acquisition and preparation. Dr Pasta also acknowledges the Italian Ministry of Education, University and Research for supporting his research.

\section{References}

1. Bonnichsen CR, Sundt TM III, Anavekar NS, Foley TA, Morris MF, Martinez MW, et al. Aneurysms of the ascending aorta and arch: the role of imaging in diagnosis and surgical management. Expert Rev Cardiovasc Ther. 2011; 9:45-61

2. Gleason TG. Heritable disorders predisposing to aortic dissection. Semin Thorac Cardiovasc Surg. 2005; 17:274-81.
3. Davies RR, Goldstein LJ, Coady MA, Tittle SL, Rizzo JA, Kopf GS, et al. Yearly rupture or dissection rates for thoracic aortic aneurysms: simple prediction based on size. Ann Thorac Surg. 2002;73:17-28.

4. Knipp BS, Deeb GM, Prager RL, Williams CY, Upchurch GR, Patel HJ. A contemporary analysis of outcomes for operative repair of type A aortic dissection in the United States. Surgery. 2007;142:524-8.

5. Rampoldi V, Trimarchi S, Eagle KA, Nienaber CA, Oh JK, Bossone E, et al. Simple risk models to predict surgical mortality in acute type A aortic dissection: the International Registry of Acute Aortic Dissection score. Ann Thorac Surg. 2007; 83:55-61

6. Coady MA, Rizzo JA, Goldstein LJ, Elefteriades JA. Natural history, pathogenesis, and etiology of thoracic aortic aneurysms and dissections. Cardiol Clin. 1999; 17:615-35, vii.

7. Johansson G. Ruptured thoracic aortic aneurysms: a study of incidence and mortality rates. J Vasc Surg. 1995;21:985-8.

8. Cripe L, Andelfinger G, Martin LJ, Shooner K, Benson DW. Bicuspid aortic valve is heritable. J Am Coll Cardiol. 2004;44:138-43. 
9. Bonderman D, Gharehbaghi-Schnell E, Wollenek G, Maurer G, Baumgartner H, Lang IM. Mechanisms underlying aortic dilatation in congenital aortic valve malformation. Circulation. 1999;99:2138-43.

10. Ward C. Clinical significance of the bicuspid aortic valve. Heart. 2000;83:81-5.

11. Larson EW, Edwards WD. Risk factors for aortic dissection: a necropsy study of 161 cases. Am J Cardiol. 1984;53:849-55.

12. Edwards WD, Leaf DS, Edwards JE. Dissecting aortic aneurysm associated with congenital bicuspid aortic valve. Circulation. 1978;57:1022-5.

13. El-Hamamsy I, Yacoub MH. Cellular and molecular mechanisms of thoracic aortic aneurysms. Nat Rev Cardiol. 2009;6:771-86.

14. Davies MJ. Aortic aneurysm formation: lessons from human studies and experimental models. Circulation. 1998;98:193-5.

15. Raghavan ML, Webster MW, Vorp DA. Ex vivo biomechanical behavior of abdominal aortic aneurysm: assessment using a new mathematical model. Ann Biomed Eng. 1996;24:573-82.

16. Sommer G, Gasser TC, Regitnig P, Auer M, Holzapfel GA. Dissection properties of the human aortic media: an experimental study. J Biomech Eng. 2008;130: 021007.

17. Purslow PP. Positional variations in fracture toughness, stiffness and strength of descending thoracic pig aorta. J Biomech. 1983;16:947-53.

18. Gdoutos EE, Schubel PM, Daniel IM. Determination of critical tearing energy of tyre rubber. Strain. 2004;40:119-25.
19. Gregory JR, Spearing SM. A fiber bridging model for fatigue delamination in composite materials. Acta Materialia. 2004;52:5493-502.

20. Vorp DA, Schiro BJ, Ehrlich MP, Juvonen TS, Ergin MA, Griffith BP. Effect of aneurysm on the tensile strength and biomechanical behavior of the ascending thoracic aorta. Ann Thorac Surg. 2003;75:1210-4.

21. Iliopoulos DC, Deveja RP, Kritharis EP, Perrea D, Sionis GD, Toutouzas K, et al Regional and directional variations in the mechanical properties of ascending thoracic aortic aneurysms. Med Eng Phys. 2009;31:1-9.

22. Phillippi JA, Klyachko EA, Kenny JP, Eskay MA, Gorman RC, Gleason TG Basal and oxidative stress-induced expression of metallothionein is decreased in ascending aortic aneurysms of bicuspid aortic valve patients. Circulation 2009;119:2498-506.

23. Phillippi JA, Eskay MA, Kubala AA, Pitt BR, Gleason TG. Altered oxidative stress responses and increased type I collagen expression in bicuspid aortic valve patients. Ann Thorac Surg. 2010;90:1893-8.

24. Holzapfel GA, Sommer G, Auer M, Regitnig P, Ogden RW. Layer-specific 3D residual deformations of human aortas with non-atherosclerotic intimal thickening. Ann Biomed Eng. 2007;35:530-45.

25. Carson MW, Roach MR. The strength of the aortic media and its role in the propagation of aortic dissection. J Biomech. 1990;23:579-88.

26. Tiessen IM, Roach MR. Factors in the initiation and propagation of aortic dissections in human autopsy aortas. J Biomech Eng-Transactions of the ASME. 1993; 115:123-5. 